\title{
The effect of outflowing water coolant with supercritical parameters on a barrier
}

\author{
Maksim Alekseev ${ }^{1, *}$, Ivan Vozhakov ${ }^{1,2}$, Sergey Lezhnin ${ }^{1,2}$, and Nikolay Pribaturin ${ }^{1}$ \\ ${ }^{1}$ Kutateladze Institute of Thermophysics SB RAS, Novosibirsk, Russia \\ ${ }^{2}$ Novosibirsk State University, Novosibirsk, Russia
}

\begin{abstract}
The outflow of supercritical coolant with different initial parameters and its impact on the barrier have been numerically simulated. Spatial and axial distributions of pressure and steam quality are presented. The force acting on the barrier at different parameters of the outflow has been calculated.
\end{abstract}

\section{Introduction}

In connection with increasing intensity in modern power plants, working pressures and temperatures may approach or even exceed critical thermodynamic parameters. It is extremely important to be able to predict scenarios of dynamic processes at sudden depressurization of the work section. In particular, of great interest is to determine impacts on various obstacles at low (wave) and large times of the energy heat carrier outflow. As it was shown in [1], under non-stationary outflow of the superheated coolant into the atmosphere, an unsteady compression wave, which may damage other elements of the power plant, is generated into the environment. There are many experimental studies of the stationary jet outflow of superheated steam and boiling coolant on the barrier under high pressure. For example, in [2] the impact of the steam jet on the obstacle and the pressure distribution over the radius on the obstacle were measured. The similarity of the stationary outflow of superheated steam and the one of the gas jet was shown. Experimental study of the stationary outflow of the boiling coolant from the pipe end on the barrier in an industrial scale was carried out in [3].

\section{Governing equations}

The present study considers the axisymmetric problem of the water coolant outflow from a high pressure vessel with the aim to obtain the characteristics of wave impact on the outer barrier and the outflow parameters. The problem is solved by numerical simulation of the system of Euler equations, the equation of continuity and energy equations for a two-phase homogeneous mixture. For calculations based on computing complex LCPFCT [4] a software code was developed for solving a wide class of problems, arising during depressurization of vessels or piping with superheated water coolant. To close the system of

\footnotetext{
*Corresponding author: alekseev@itp.nsc.ru
} 
equations it is required to set the equation of state of thermodynamically equilibrium twophase mixture in the form of

$$
p=F(\rho, E)
$$

Here $\rho$ is the density, $p$ is the pressure, and $E$ is the specific internal energy.

Density, internal energy, other thermodynamic parameters and potentials of the mixture in equilibrium depend on pairs of variables, e.g. $X$ and $p$ [5].

$$
\begin{gathered}
\frac{1}{\rho}=(1-X) \frac{1}{\rho_{1}(p)}+X \frac{1}{\rho_{2}(p)} \\
H=(1-X) H_{1}(p)+X H_{2}(p), E=(1-X) E_{1}(p)+X E_{2}(p)=H-p / \rho
\end{gathered}
$$

Here $H$ is the specific enthalpy of the mixture, $X$ is the steam quality, and $L$ is the latent heat of vaporization. Index 1 refers to liquid, and 2 refers to vapor.

Using the expression (3) and the equality $L=H_{2}-H_{1}$, it is possible to obtain

$$
E=(1-X) E_{1}+X E_{2}=H-p / \rho=H_{1}+X L-p / \rho
$$

Since from (2) follows

$$
X(p, \rho)=\frac{1 / \rho-1 / \rho_{1}(p)}{1 / \rho_{2}(p)-1 / \rho_{1}(p)},
$$

then, from (4) we obtain the equation of state of the two-phase mixture in the form of relationship between pressure, density and specific internal energy:

$$
E(p, \rho)=H_{1}(p)-\frac{\left.L(p) / \rho_{1} p\right)}{1 / \rho_{2}(p)-1 / \rho_{1}(p)}+\left[\frac{L(p)}{1 / \rho_{2}(p)-1 / \rho_{1}(p)}-p\right] \cdot \frac{1}{\rho}
$$

From equation (6) the dependence (1) was numerically tabulated in advance to further use in the numerical simulation. Calculation of properties of steam and water in sub- and supercritical states was performed using the software package TTSE [6].

\section{Modeling results and discussion}

In the present work, the outflow of water with different initial supercritical parameters on the barrier was numerically simulated. On the PV-diagram with initial $P_{0}=23 \mathrm{MPa}$ and $T_{0}=370{ }^{\circ} \mathrm{C}$, the adiabatic line passes to the left of the critical point and crosses the line of boiling, i.e. enters the two-phase region with void fraction equal to null [7]. In the case when the initial parameters take values $P_{0}=23 \mathrm{MPa}$ and $T_{0}=380^{\circ} \mathrm{C}$, the adiabatic line passes to the right of the critical point and crosses the line of condensation, i.e. enters the twophase region with void fraction equal to unity.

Figure 1 presents spatial distributions of normalized pressure gradient and steam quality. In the initial moment of time at depressurization, the generation of compression waves occurs. The compression wave moves towards the obstacle and is reflected from it. Behind the compression wave, forms the area of the two-phase mixture outflow, consisting of the Mach disk and oblique shock wave. The shape of the Mach disk and the lateral suspension surges differ for the cases of evaporation and condensation. At vapor outflow with condensation, the form of the Mach disk and the lateral suspension surges are similar to the form, arising at a gas-dynamic outflow. At liquid outflow with evaporation, the shape of the lateral suspension surges with the Mach disk approaches hemispherical.

Figures 2, 3 show axial profiles of pressure and steam quality at various points in time. The vertical solid line shows the coordinate of the target, and the vertical dotted line shows the coordinate of the channel end. The amplitudes of compression waves at propagation and reflection from the obstacle are similar for the process of evaporation and condensation. 
Together with the compression wave, the rarefaction wave moves in the interior of the channel. From the drawings it is seen that the propagation velocity of the condensation
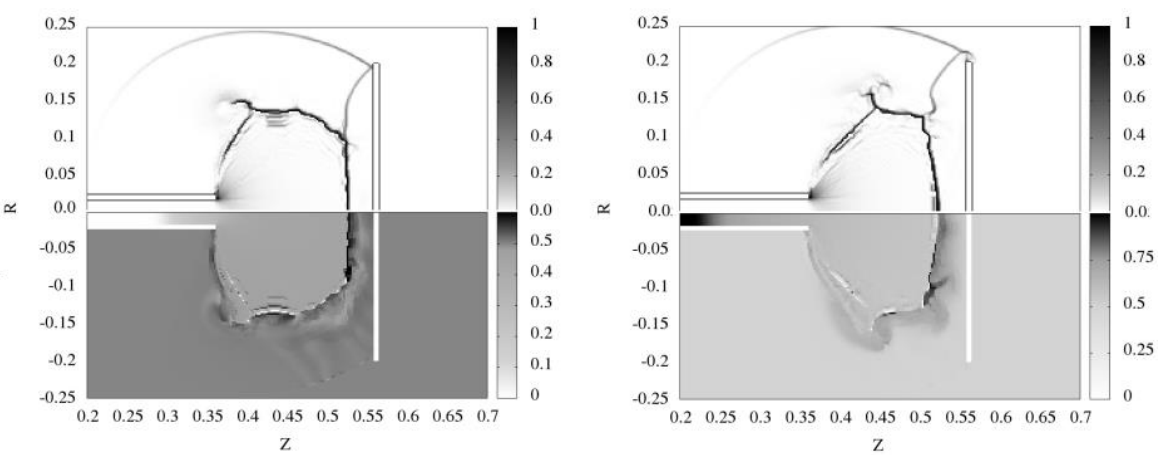

Fig. 1. Spatial distribution of normalized pressure gradient (top) and steam quality (bottom) at time $\mathrm{t}=0.5 \mathrm{~ms}$ for initial conditions $P_{0}=23 \mathrm{M \Pi a}, \mathrm{T}_{0}=370{ }^{\circ} \mathrm{C}$ (left) and $T_{0}=380{ }^{\circ} \mathrm{C}$ (right).

front is higher than that of the evaporation front inside the channel. The main change of steam quality in case of condensation occurs inside the channel, and for the case of evaporation, the phase transition continues outside of the channel as well. In addition, the case of condensation is characterized by a sharp pressure drop at the end channel, while for the case with evaporation, this pressure drop is not observed.
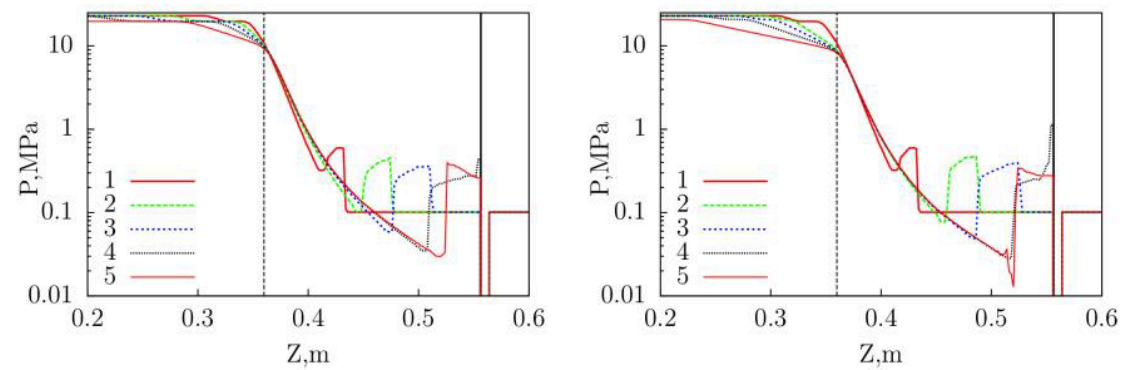

Fig. 2. Axial pressure distribution in different moments of time $(1-0.09 \mathrm{~ms}, 2-0.15 \mathrm{~ms}, 3-0.21$ $\mathrm{ms}, 4-0.3 \mathrm{~ms}$, and $5-0.51 \mathrm{~ms}$ ) for initial conditions $P_{0}=23 \mathrm{M \Pi a}, T_{0}=370{ }^{\circ} \mathrm{C}$ (left) and $T_{0}=380{ }^{\circ} \mathrm{C}$ (right). Solid vertical line is the coordinate of the target, and the dotted vertical line is the coordinate of the channel end.
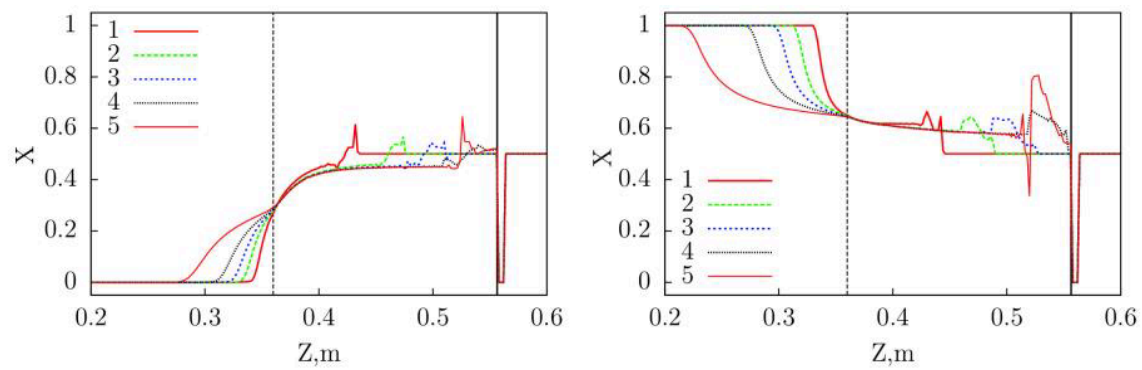

Fig. 3. Axial distribution of steam quality at different points in time $(1-0.09 \mathrm{~ms}, 2-0.15 \mathrm{~ms}, 3-$ $0.21 \mathrm{~ms}, 4-0.3 \mathrm{~ms}$, and $5-0.51 \mathrm{~ms}$ ) for initial conditions $P_{0}=23 \mathrm{MPa}, T_{0}=370{ }^{\circ} \mathrm{C}$ (left) and $T_{0}=380$ ${ }^{0} \mathrm{C}$ (right). Solid vertical line is the coordinate of the target, and the dotted vertical line is the coordinate of the channel end. 
Figure 4 shows the time dependence of the force, acting on the barrier and appearing during the water coolant outflow. The differences between the cases of evaporation and condensation are due to different velocities of perturbation waves' propagation in the external space (time shift). In addition, it is seen that the maximum value of the force acting on the barrier is reached at time moments $0.45 \mathrm{~ms}\left(T_{0}=380{ }^{\circ} \mathrm{C}\right)$ and $0.5 \mathrm{~ms}\left(T_{0}=370{ }^{\circ} \mathrm{C}\right)$, that is followed by fall.

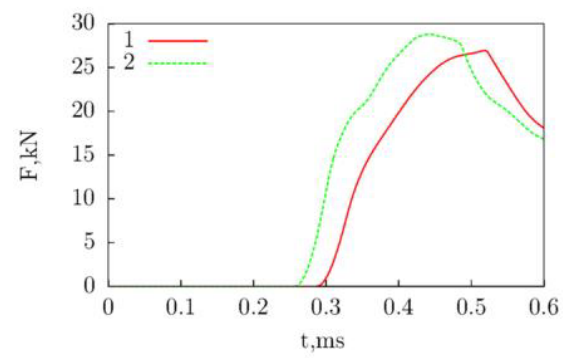

Fig. 4. Time dependence of the force acting on the barrier at outflow of water in supercritical state. 1$P_{0}=23 \mathrm{MPa}, T_{0}=370{ }^{\circ} \mathrm{C}, 2-P_{0}=23 \mathrm{MPa}, T_{0}=380{ }^{\circ} \mathrm{C}$.

\section{Conclusion}

The outflow of water with initial supercritical parameters has been numerically simulated using the equilibrium model of phase transition. It is shown that, depending on initial parameters, there are various options for the outflow development. The options are associated with the presence of the "opposite" phase transitions: evaporation or condensation. At steam outflow with condensation, the form of the Mach disk and the lateral suspension surges are similar to the form, arising at gas-dynamic outflow. At the liquid outflow with evaporation, the shape of the lateral suspension surges with a Mach disk approaches hemispherical. The results of the calculation of the spatial and axial distribution of pressure and steam quality are presented. It is noted that in the case of condensation, phase transition occurs mainly within the channel, and in the case of evaporation, it takes place both inside and outside of the channel. Forces acting on the barrier are calculated at different initial parameters.

The work was financially supported by the Russian Science Foundation (grant No14-29-00093).

\section{References}

1. G.V. Gofman, A.E. Kroshilin, B.I. Nigmatulin, High Temp. 19897 (1981)

2. F. Masuda, Nuclear Eng. Design 67273 (1982)

3. T. Isozaki, S. Miyazono, Nuclear Eng. Design 961 (1986)

4. J.P. Boris, A.M. Landsberg, E.S. Oran, J.H. Garder, Naval Research Lab Washington DC (1976)

5. M.D. Weisman, Thermodynamics of Vapor-Liquid Flows (1967)

6. Guideline on the TTSE method for calculation of thermodynamic properties of water and steam applied to IAPWS-95 (2003)

7. I.S. Vozhakov, M.V. Alekseev, S.I. Lezhnin, N.A. Pribaturin, J. Phys.: Conference Series 754032021 (2016) 